\title{
Self-plasticizing Membrane Based on Co(II)-porphyrin Modified with Silver Nanoparticles for Thiocyanate Detection
}

\author{
Siti Nuraqidah Zainal, ${ }^{1,2}$ Majid Rezayi, ${ }^{1,2}$ \\ Yatimah Alias, ${ }^{1,2^{*}}$ and H. N. M. Ekramul Mahmud ${ }^{1 * *}$ \\ ${ }^{1}$ Department of Chemistry, Faculty of Science, University of Malaya, Kuala Lumpur 50603, Malaysia \\ ${ }^{2}$ University Malaya Centre for Ionic Liquids (UMCiL), University of Malaya, Kuala Lumpur 50603, Malaysia
}

(Received April 5, 2019; accepted July 12, 2019)

Keywords: self-plasticizing polymeric membrane, Co(II)-porphyrin, silver, thiocyanate, sensor

A novel potentiometric anion-selective self-plasticizing polymeric membrane based on 5,10,15,20-tetrakis-(4-methoxyphenyl)-21H,23H-porphyrin cobalt(II) (CoTMeOPP)-silvernanoparticle-modified glassy carbon electrode (GCE) was examined for use in the detection of thiocyanate anions. The membrane was characterized by X-ray diffraction (XRD) analysis, field-emission electron microscopy, UV-visible diffused reflectance spectroscopy, and fluorescence spectroscopy. The prepared ion-selective sensor exhibited a positive Nernstian response and deviated from the classical Hofmeister selective pattern with a significantly sensitive and enhanced response towards the thiocyanate anion. It exhibited a rapid response of $3 \mathrm{~s}$ with a low detection limit of $9.55 \times 10^{-8} \mathrm{M}$ within the concentration range from $1 \times 10^{-1}$ to $1 \times 10^{-7} \mathrm{M}$. The sensor also showed a high selectivity for the thiocyanate anion over a variety of anions. The influence of the additives was studied and this sensor was successfully applied to the potentiometric titration of silver nitrate and the direct determination of the amount of thiocyanate anions in a wide array of samples of river water, ex-mine lake sediment, human saliva, and fish.

\section{Introduction}

Trace amounts of thiocyanate infiltrates the human body via food and medicines but mainly from cigarettes. It is then excreted out of the body through saliva, urine, and serum after being processed by the kidneys. ${ }^{(1)}$ Thiocyanate serves many purposes in industry and widely used in various applications. As a result, trace amounts of thiocyanate is present in the environment. ${ }^{(2)}$ An elevated amount of thiocyanate causes cell self-destruction and iodine deficiency in the body, delays neurological functional development of infants, reduces the production of thyroid hormone, and causes chronic changes in liver, kidney, and muscle cells when it is absorbed in the blood. ${ }^{(3)}$ Several analytical methods for the quantitative determination of thiocyanate have been reported, including amperometry methods, ion chromatography (IC), high-performance gas and liquid chromatography (HPLC), atomic absorption spectrometry (AAS), and fluorescence spectrometry. ${ }^{(4)}$ Despite the low detection limit and wide working range of the

\footnotetext{
*Corresponding author: e-mail: yatimah70@um.edu.my

** Corresponding author: e-mail: ekramul@um.edu.my

https://doi.org/10.18494/SAM.2019.2393
} 
reported methods, they involve costly instruments, require sophisticated operating skills, must be conducted in the laboratory, and are time-consuming and tedious. ${ }^{(5)}$

Ion-selective electrodes (ISEs) have emerged as a type of tool to minimize the cut breaks of the prior detection methods and have been proven to have fast response and high selectivity as well as sensitivity over a wide dynamic range. ${ }^{(6)}$ Anion-selective membrane electrodes using ionophores as the active material were proven to deviate from the classic Hofmeister selectivity sequence, i.e., $\mathrm{ClO}^{-}>\mathrm{SCN}^{-}>\mathrm{I}^{-}>\mathrm{NO}^{-}>\mathrm{Br}^{-}>\mathrm{NO}^{-}>\mathrm{Cl}^{-}$. Several ionophores have been found to alter the Hofmeister order and metalloporphyrins are one of them. ${ }^{(7,8)}$ Metalloporphyrins have been extensively used by numerous researchers for at least two decades since they deviated from the classical Hofmeister selectivity sequence, can be tailored for different sensitivities towards specific anions or cations by introducing different metals to the porphyrin rings, and they satisfy most of the desired and relevant ionophore qualities. The ionophore of 5,10,15,20-tetrakis-(4-methoxyphenyl)-21H,23H-porphyrin cobalt(II) (CoTMeOPP) can be used with any common functional group and solvent without being affected by the catalytic activities of the polar groups. ${ }^{(9)}$ It is a versatile ligand that forms welldefined complexes with many types of different metals and offers many other qualities. ${ }^{(10)}$ Tetraphenylporphyrin (TPP) is among the first synthetic porphyrins to be electrochemically investigated. The electrochemistry of this compound is similar to those of almost all compounds, i.e., stepwise oxidized or stepwise reduced by two electrons at the $\pi$-ring system to give $\pi$-cation radicals. ${ }^{(11)}$ Metalloporphyrins, as mentioned earlier, have many qualities that can be tailored for use as ionophores for detecting the target analyte, i.e., thiocyanate anions. The introduction of cobalt (II) $\left(\mathrm{Co}^{2+}\right)$ to the porphyrin ring involves both a metal-centered process, i.e., the incorporation of $\mathrm{Co}$ (II) in the porphyrin ring, which influences the site of electron transfer of an ionophore, and a ring-centered process, i.e., reversible first ring-centered oxidation and first ring-centered reduction, which make it selective and sensitive towards thiocyanate anions. ${ }^{(12)}$

There are many reported works on the detection of thiocyanate using different ionophores as the active material. Recently, (N-3-methyl phenyl salicylidenaminato)-copper(II) ${ }^{(13)}$ 2'-[(4,5dimethyl-1,2-phenylene) bis[(E)-nitrilomethylidyne]] bis [4,6-bis (1,1-dimethylethyl) with $\mathrm{Mn}(\mathrm{III})$-salophen chloride], ${ }^{(14)}$ and sulfadimidine metal complexes ${ }^{(15)}$ were utilized as ionophores in sensors for the detection of thiocyanate. The main composition of ISEs normally consists of an electrode and ion-selective membranes (ISMs), which consist of ionophores, additives, monomers, and plasticizers. ${ }^{(16)}$ Many researchers reported that thiocyanate sensors worked effectively with polyvinyl chloride (PVC), ${ }^{(13,17)}$ polyaniline (PANi), and acrylate polymeric membranes. ${ }^{(18)}$ PVC has been widely used in the fabrication of sensors. The utilization of PVC as the polymeric membrane in sensor fabrication requires many different types of plasticizer such as dibutyl sebacate (DBS), di-n-butyl phthalate (DBP), and 2-nitrophenyloctylether ( $o$-NPOE). Plasticizers have been proven to be toxic to the environment. The utilization of a plasticizer in the fabrication of sensors comes with many impediments, i.e., it leads to the exudation and leaching of plasticizers from the membrane to the environment and reduces the sensor lifetime. ${ }^{(6)}$ The leaching of plasticizers weakens the adhesion of the polymeric membrane onto the glassy carbon electrode (GCE). Also, the reported toxicity of the commercially used plasticizers to the environment will have many drawbacks in the future, especially the pollution of the environment by these highly toxic chemicals. ${ }^{(19)}$ 
Self-plasticizing polymeric membranes are emerging as alternatives to curb the problems of leaching and the utilization of plasticizers. Many types of polymeric membranes have been explored as self-plasticizing polymeric membranes for the fabrication of sensors to avoid the utilization of plasticizers. In recent years, there are new types of alternative self-plasticizing polymeric membrane to replace PVC, namely, polyurethane (PU), silicon rubber, PANi, and acrylate. ${ }^{(20)}$ Acrylate membranes have relatively low $\mathrm{T} g$ with photocurable properties that enable self-polymerization simply by exposure to UV-Vis. Therefore, the utilization of acrylate polymers excludes the use of plasticizers and hence eliminates the drawbacks of plasticizers and increases the performance of ISEs. ${ }^{(6)}$ A silver (Ag) layer was introduced as the foundation layer between GCE and the polymeric membrane to increase the synergistic effect between the GCE and the active compounds of the sensor on the detection of the analyte. Consequently, the performance of the self-polymerized thiocyanate sensor was enhanced.

In this study, for the first time, CoTMeOPP has been used as the ionophore and fabricated as a polymeric membrane with self-plasticizing hydroxyl-ethyl methacrylate (HEMA) and $n$-butyl acrylate (n-BA) to overcome the stumbling block of the use of plasticizers in the ISM composition of sensors for the detection of thiocyanate. The thiocyanate-selective membranes were enhanced by introducing a Ag layer prior to the deposition of the first and second membranes onto the GCE for better selectivity and sensitivity of the sensor. Detection using the self-plasticizing thiocyanate ISE sensors is based on an indirect method of measuring trace amounts of thiocyanate. The GCE was chosen as the ISE for its advantages such as its comparatively fast response, high selectivity with a wide dynamic range, and low cost. The thiocyanate-selective self-plasticizing membranes were prepared, optimized, and checked at different concentrations of thiocyanate anions and then used to determine the thiocyanate concentration in pure solutions, river water, and soil sediments.

\section{Experimental Procedure}

\subsection{Reagents}

Membrane materials such as CoTMeOP as the ionophore, 1,6-hexanediol di-acrylate (HDDA) as the cross-linker, 2,2-dimethoxy-2-phenylacetophenone (DMPP) as the initiator, and tetradodecylammoniumchloride (TDDA) as the additive were purchased from Sigma-Aldrich (USA). HEMA and n-butyl acrylate (n-BA) were purchased from Merck (Germany) and used as monomers. Silver nitrate was purchased from Merck (USA). Potassium chloride was purchased from Lobal Chemie (India). Acetone, methanol, and DMSO solvents were purchased from R\&M Chemicals (U.K.). Potassium thiocyanate and all other chemical salts of analytical grade were purchased from Sigma-Aldrich (USA) and Fluka (USA). Deionized and distilled water were used to prepare all aqueous solutions.

\subsection{Electrodeposition of Ag-nanoparticle-modified GCE}

Silver nanoparticles (Ag NPs) were electrodeposited by cyclic voltammetry (CV) onto the surface of polished GCE. An aqueous solution of $2 \times 10^{-3} \mathrm{M} \mathrm{AgNO}_{3}$ containing $1 \mathrm{M} \mathrm{KNO}_{3}$ at 
a $\mathrm{pH}$ of 5.9 was scanned from -0.08 to $-0.50 \mathrm{~V}$ at a rate of $50 \mathrm{mV} / \mathrm{s}$ for 5 cycles. The polished $\mathrm{GCE}, \mathrm{Ag} / \mathrm{AgCl}$, and platinum wire were used as the working, reference, and counter electrodes, respectively.

\subsection{Preparation of membrane electrode-Ag-NP-modified GCE}

The Ag-NP-modified GCE was soaked in $0.01 \mathrm{M} \mathrm{KCl}$ at a $\mathrm{pH}$ of 6.8 for $15 \mathrm{~min}$ and dried at room temperature. A homogenous mixture consisting of $95.14 \mathrm{wt} \%$ HEMA as the monomer and $4.86 \mathrm{wt} \%$ DMPP as the photoinitiator was prepared by sonication. $0.50 \mu \mathrm{L}$ of this mixture was drop-cast onto the Ag-NP-modified GCE and was polymerized in a UV box with a constant flow of nitrogen gas for $480 \mathrm{~s}$. The polymerized inner layer of the membrane was then dipped into $0.10 \mathrm{M}$ potassium thiocyanate $(\mathrm{KSCN})$ for $15 \mathrm{~min}$. The outer layer of the membrane was prepared by mixing $0.34 \mathrm{wt} \%$ CoTMeOPP, $0.56 \mathrm{wt} \%$ DMPP, $0.08 \mathrm{wt} \%$ HDDA, $0.16 \mathrm{wt} \% \mathrm{TDDA}$, and $98.86 \mathrm{wt} \% n$-BA. $0.50 \mu \mathrm{L}$ of the as-prepared mixture was then drop-cast onto the hydrated poly-(HEMA) inner layer and polymerized in a UV box with a constant flow of nitrogen gas for $480 \mathrm{~s}$.

\subsection{Characterization of thiocyanate (SCN)-selective membrane}

The membrane was characterized by X-ray diffraction (XRD) (Empyrean) analysis, scanning electron microscopy (SEM), and energy-dispersive X-ray spectroscopy with field electron scanning electron microscopy (FESEM) (EG Quanta 450, EDX Oxford). The electromagnetic force (EMF) measurements of fabricated sensors were performed relative to a $\mathrm{Ag} / \mathrm{AgCl}$ double-junction reference electrode using a digital $\mathrm{pH} / \mathrm{ISE}$ meter (Orion Star A214). The GCE as the working electrode and $\mathrm{Ag} / \mathrm{AgCl}$ as the reference electrode were purchased from Bioanalytical Systems, Inc. (BASi) USA, and platinum as the counter electrode was purchased from Metrohm. CV was performed using a potentiostat/galvanostat (Autolab PG-STAT$302 \mathrm{~N}$, Metrohm). The self-plasticizing photoinitiated membranes were subjected to UV LED irradiation in an exposure box $(220 / 240 \mathrm{~Hz})$ for $500 \mathrm{~s}$ with continuous purging by $\mathrm{N}_{2}$ gas. The spectrophotometer UV/Vis/NIR (UV-3600 Shimadzu) with the temperature controller TCC2 and fluorescence spectrophotometer (AvaSpec-2048 XL) were used for characterizing the samples. All the experiments were conducted at room temperature.

\section{Results and Discussion}

\subsection{Physicochemical characterization}

\subsubsection{Cyclic voltammograms}

Figure 1(a) shows the structure of the CoTMeOPP ionophore used in this study. The introduction of $\mathrm{Co}$ (II) metal to the porphyrin rings causes them to become metalloporphyrin, giving rise to the sensitivity and selectivity of the ionophore to $\mathrm{SCN}^{-}$anions. Figure 1(b) shows 

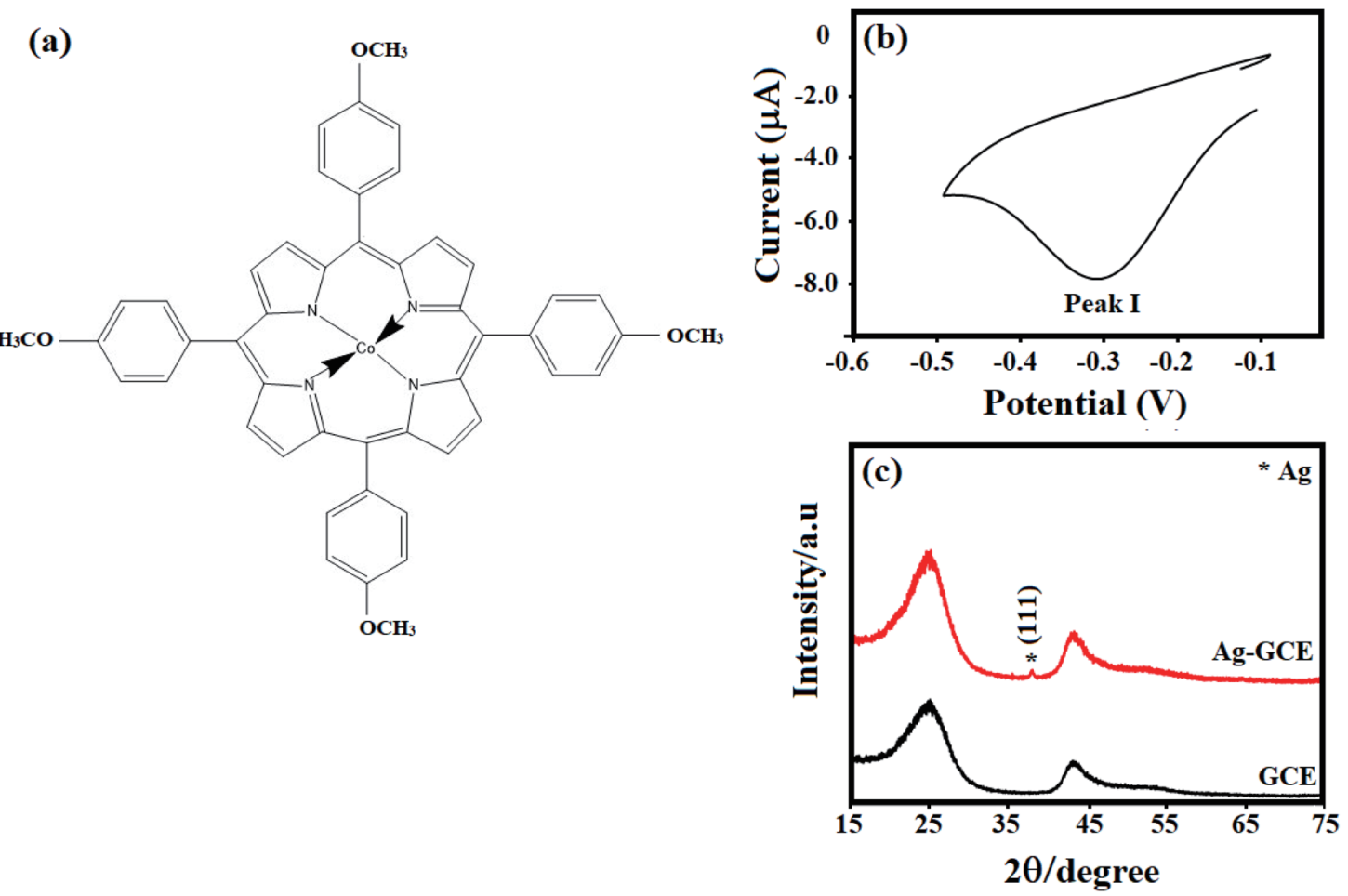

Fig. 1. (Color online) (a) Structure of 5,10,15,20-tetrakis-(4-methoxyphenyl)-porphyrin-Co(II) (CoTMeOPP), (b) first-cycle cyclic voltammogram of GCE in $1 \mathrm{M} \mathrm{KNO}_{3}$ solution containing $2 \mathrm{mM} \mathrm{AgNO}_{3}$, and (c) XRD patterns of GCE and Ag deposited onto GCE.

a cyclic voltammogram of the Ag NPs electrodeposited onto the surface of GCE. As seen, the cathodic peak at $-0.29 \mathrm{~V}$ (peak I) observed in the first scan corresponds to the $\mathrm{Ag}^{+}+\mathrm{e}^{-} \leftrightarrow$ $\mathrm{Ag}(\mathrm{s})$ reaction, i.e., the deposition of Ag NPs on the GCE. The cathodic peak current represents the typical process of Ag deposition on GCE.

\subsubsection{Crystal characterization}

The XRD pattern of bare GCE and Ag-NP-modified GCE are shown in Fig. 1(c). As seen in Fig. 1(c), the main feature of the XRD pattern of the Ag peak at $2 \theta$ of around $38^{\circ}$ can be seen clearly compared with the XRD pattern of bare GCE. This significant peak is due to the Bragg reflections corresponding to the (111) set of Ag planes. The significant peak can be indexed to the FCC structure of Ag deposited onto the GCE. The interplanar distance, i.e., d-spacing, of the deposited Ag was determined using Bragg's equation; d[111] of Ag was $0.236 \mathrm{~nm}$.

\subsubsection{Morphology and chemical characterization}

The morphology of Ag deposited onto GCE was characterized by FESEM, as shown in Fig. 2. The low-magnification FSEM image in Fig. 2(a) clearly shows the homogenous formation of Ag 


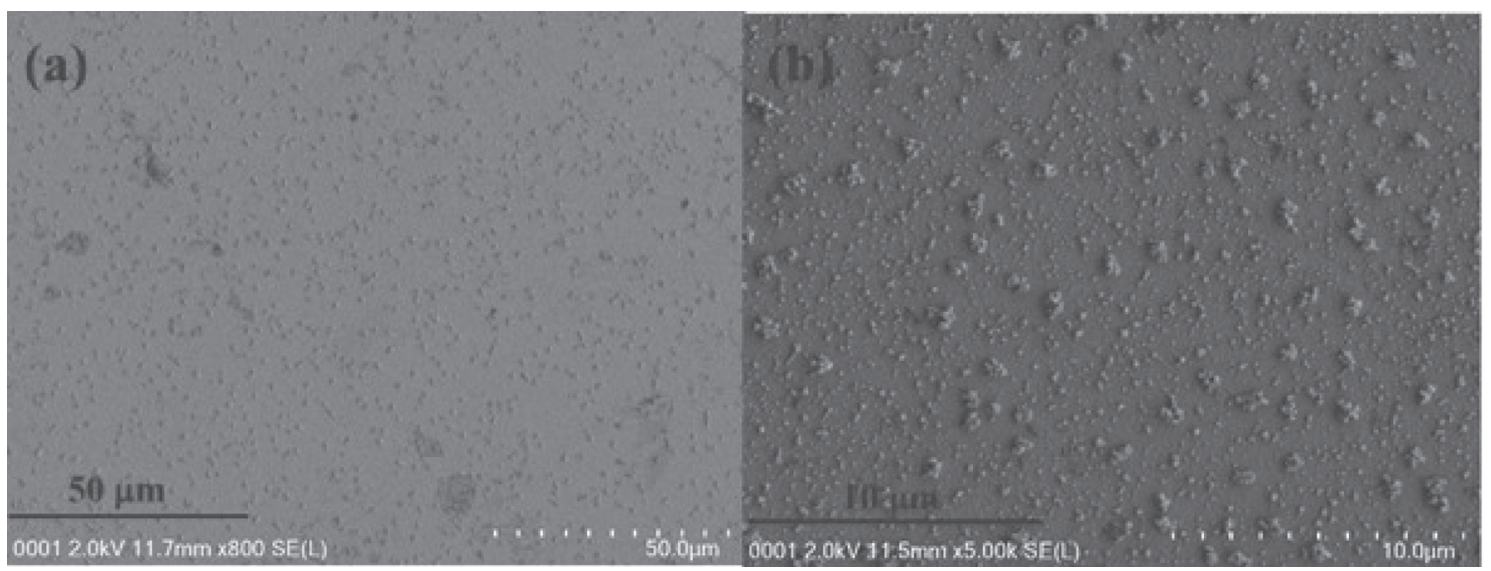

Fig. 2. (Color online) (a) Low-magnification and (b) high-magnification FESEM images of Ag NPs deposited onto GCE.

nanoparticles onto the surface of GCE. The high-magnification FESEM image shown in Fig. 2(b) shows a number of Ag NPs agglomerated together owing to the high electrostatic force, i.e., large surface-to-volume ratio, between the Ag NPs. ${ }^{(21)}$

To investigate the formation and growth of the self-plasticizing $\mathrm{SCN}^{-}$-selective membrane on the GCE, different layers of the membrane were observed by FESEM and energy dispersive X-ray (EDX) analysis. Figure 3 depicts the FESEM images and EDX results of the selfpolymerized inner membrane and the optimized $\mathrm{SCN}^{-}$-selective membrane. It can be seen clearly in Fig. 3(a) that crystalline Ag NPs were dispersed and embedded underneath the smooth layer of the HEMA polymer, whereas Fig. 3(b) shows the morphology of the $\mathrm{SCN}^{-}$selective membrane. It can be seen that the $\mathrm{SCN}^{-}$-selective membrane has distinctive crystalline geometrical structures that are closely packed and stacked together with CoTMeOPP ionophores. EDX results in Fig. 3(c) show the elemental peak of Ag at $2.91 \mathrm{keV}$, confirming the presence of Ag deposited onto the GCE. The peaks of Co and other components can also be seen in the EDX spectra in Fig. 3(d), supporting the presence and successful deposition of Co and other active components of the composite onto GCE.

\subsubsection{Optical properties}

The complexation of CoTMeOPP ionophores and $\mathrm{SCN}^{-}$ions was examined spectrophotochemically. Solutions of $2 \times 10^{-4} \mathrm{M}$ CoTMeOPP and $1 \mathrm{mM} \mathrm{SCN}^{-}$were prepared by dissolving each of CoTMeOPP and $\mathrm{SCN}^{-}$in dimethyl sulfoxide (DMSO) solvent and mixed at a 1:10 (v/v) ratio to obtain a mixture of $\mathrm{CoTMeOPP}$ ionophores and $\mathrm{SCN}^{-}$solutions. The UV spectra of CoTMeOPP, $\mathrm{SCN}^{-}$, and their mixture (1:1, v/v) were recorded over 450-600 nm. ${ }^{(22)}$ The UV-Vis spectrum of the CoTMeOPP ionophore in Fig. 4(a) shows a significant peak at $537 \mathrm{~nm}$. The peak at $537 \mathrm{~nm}$ is the distinctive porphyrin peak indicating the Q-band 

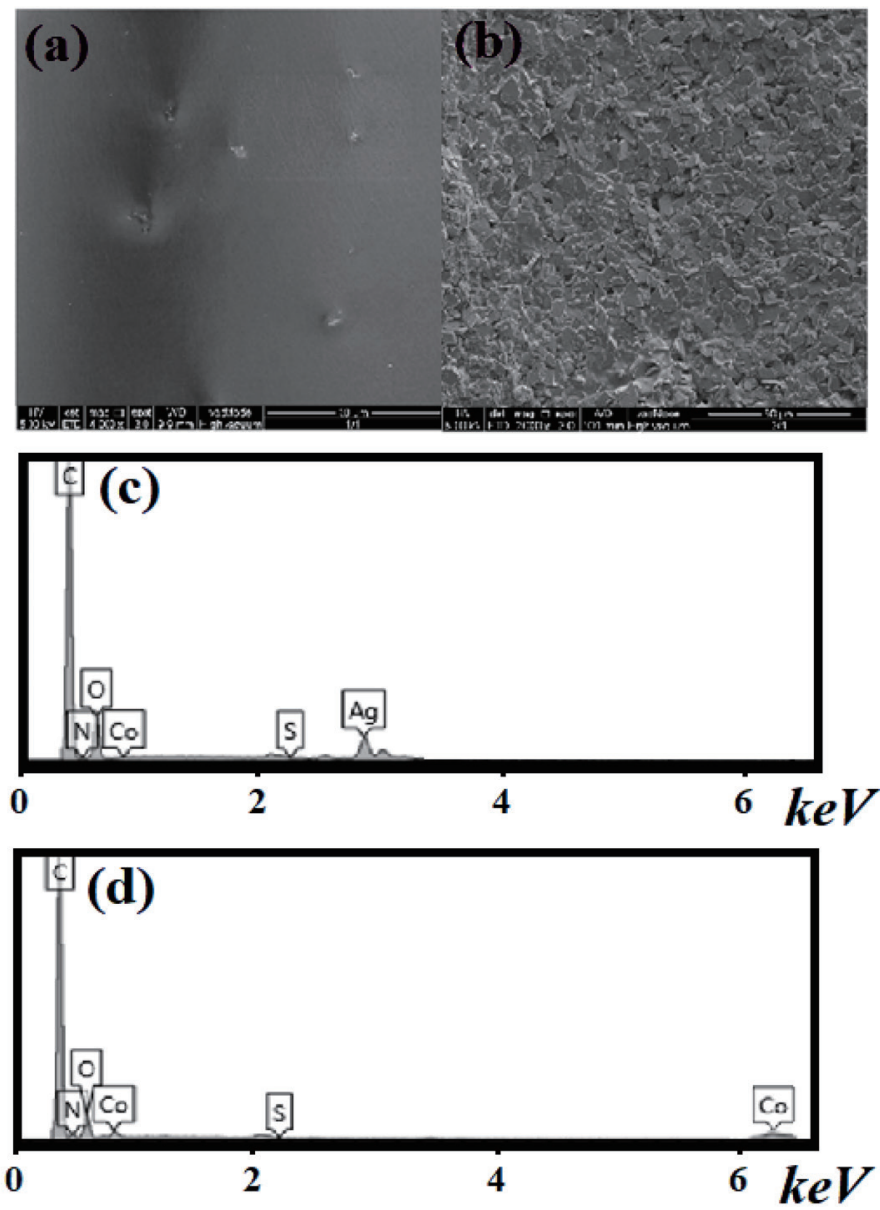

Fig. 3. (Color online) FESEM images of (a) inner layer and (b) outer layer of the self-polymerized $\mathrm{SCN}^{-}$-selective membrane. EDX spectra of (c) inner layer and (d) outer layer of the membrane.

of free porphyrin in solution with the transition of $\mathrm{a}_{2 \mathrm{u}}(\pi) \rightarrow \mathrm{e}_{\mathrm{g}}{ }^{*}(\pi)$. The spectrum of $\mathrm{SCN}^{-}-$ CoTMeOPP in Fig. 4(a) shows a significant decrease in the intensity of the peak. The decrease in the peak indicates the decrease in free $\mathrm{Co}^{3+}$ and free porphyrin contents, ${ }^{(23)}$ and this may be due to the complexation occurring between free Co metal and $\mathrm{SCN}^{-}$anions. The number of absorption peaks does not change after CoTMeOPP is coordinated with $\mathrm{SCN}^{-}$, indicating that after $\mathrm{SCN}^{-}$is coordinated with the central metal, the symmetry group of $\mathrm{CoTMeOPP}-\mathrm{SCN}^{-}$ agrees with the $\mathrm{C}_{2 \mathrm{v}}$ symmetry group.

To study the stoichiometry of the complexes formed from CoTMeOPP ionophores and $\mathrm{SCN}^{-}$ anions, titration between $2.5 \mathrm{ml}$ of $2.3 \times 10^{-6} \mathrm{M}$ CoTMeOPP ionophores and $1.0 \times 10^{-4} \mathrm{M}$ $\mathrm{SCN}^{-}$anions in DMSO solvent at $25{ }^{\circ} \mathrm{C}$ was conducted. The UV-Vis spectra were recorded in the range of 200 to $800 \mathrm{~nm}$ for 2 min after each addition, as shown in Fig. 4(b). The maximum absorbance at $416 \mathrm{~cm}^{-1}$ was chosen to plot absorbance versus the molar ratio of 

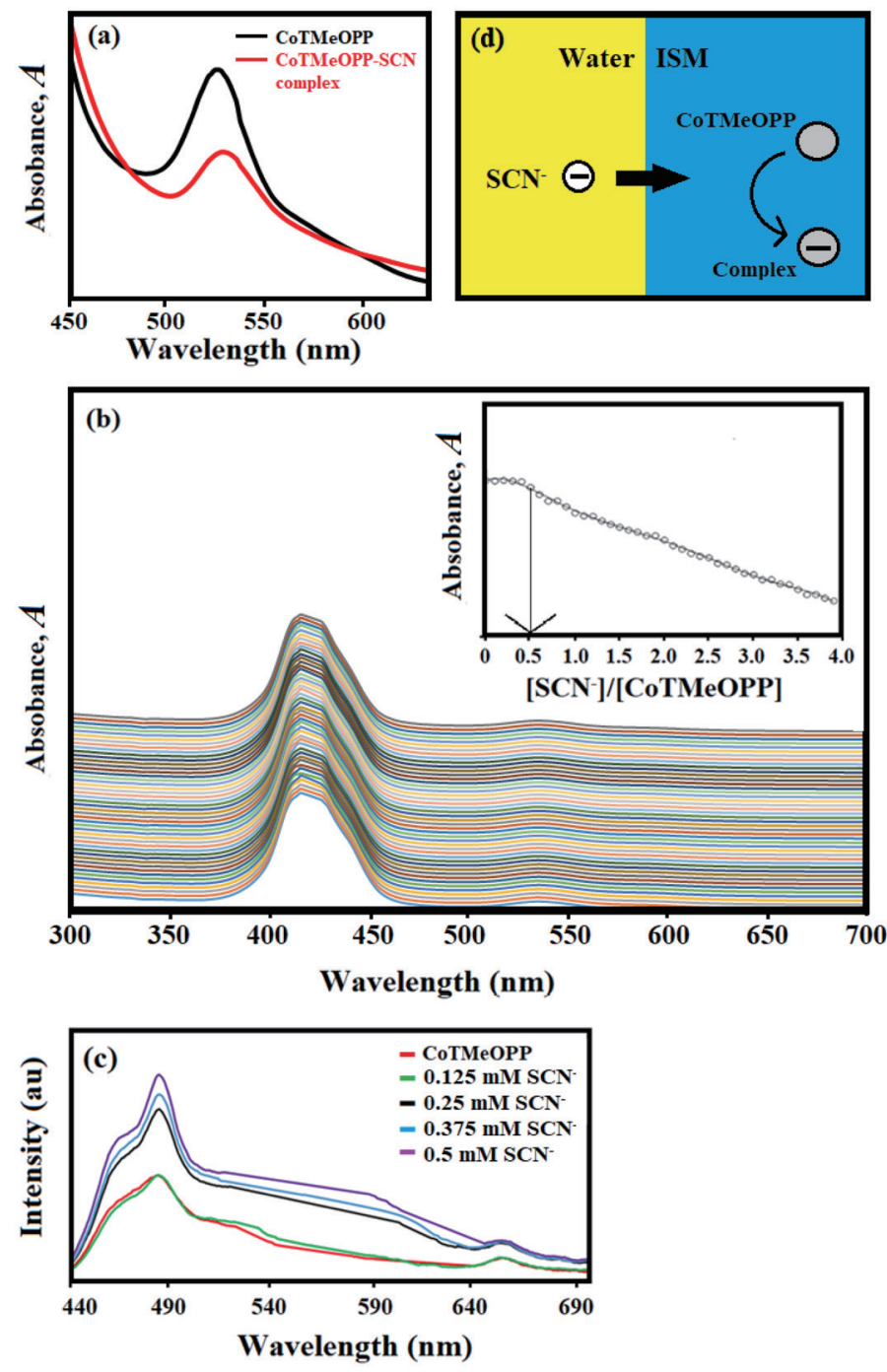

Fig. 4. (Color online) (a) UV-Vis spectra of $2.0 \times 10^{-4} \mathrm{M}$ CoTMeOPP and mixture of ionophores and $\mathrm{SCN}^{-}$ anions $(1: 10, \mathrm{v} / \mathrm{v}),(\mathrm{b}) \mathrm{UV}-\mathrm{Vis}$ absorption spectra of CoTMeOPP ionophores $\left(2.3 \times 10^{-6} \mathrm{M}\right)$ in 1:1 DI:DMSO with increasing concentration of $\mathrm{SCN}^{-}$, (c) fluorescence emission spectra of $\mathrm{CoTMeOPP}$ with the addition of 0.5, 1.0, 1.5 , and $2 \mathrm{ml}$ of KSCN. (d) Schematic diagram of electrochemical mechanism based on the formation of the target $\mathrm{SCN}^{-}$anion followed by complexation with an ionophore.

$[\mathrm{SCN}] /[\mathrm{COTMeOPP}]$ and it shows a breaking point at 0.5 as shown in Fig. $4(\mathrm{~b})$. It manifested the formation of $2: 1\left[\mathrm{CoTMeOPP}: \mathrm{SCN}^{-}\right]$of the ionophore to the target anion when the complexes are formed. Thus, the proposed general mechanism for the complexation is

$$
2 \mathrm{CoTMeOPP}+\mathrm{SCN}^{-} \leftrightarrow \mathrm{SCN}(\mathrm{CoTMeOPP})_{2}
$$

The complexation of CoTMeOPP and $\mathrm{SCN}^{-}$is also studied by fluorescence spectroscopy 
by dissolving both $1 \times 10^{-4} \mathrm{M} \mathrm{CoTMeOPP}$ and $1 \times 10^{-2} \mathrm{M} \mathrm{SCN}^{-}$in methanol. The tetrakis (4-hydroxyphenyl) porphyrin derivatives are fluorescent and in the solid state, the ligand, i.e., Co(II), shows a strong emission in the range of 484-485 $\mathrm{nm}$ upon excitation, as shown in Fig. 4(c). Another small fluorescence emission peak corresponding to $\mathrm{Co}(\mathrm{II})$ at $655 \mathrm{~nm}$ resulted from the presence of metalloporphyrin, which causes quenching, in the ionophore. The central metal ions in the complex, $\mathrm{Co}(\mathrm{II})\left(\mathrm{d}^{7}\right)$, are in the +2 oxidation state and are paramagnetic. Thus, the fluorescence intensity is low. The increase in fluorescence emission peak intensity with each subsequent addition of $\mathrm{SCN}^{-}$clearly shows the interaction between CoTMeOPP and $\mathrm{SCN}^{-}$ owing to the complexation between the CoTMeOPP ionophore and $\mathrm{SCN}^{-}$, which increases the intensity of the fluorescence emission spectra as it restores the TPP fluorescence property of the CoTMeOPP ionophore. Using the complexation results obtained, we here propose a hypothetical mechanism based on the formation of a complex between the CoTMeOPP ionophore and the $\mathrm{SCN}^{-}$target anion as shown in Fig. 4(d).

\subsection{Sensor}

\subsubsection{Potential responses}

In preliminary experiments, the CoTMeOPP ionophore was used as the active material in the construction of ion-selective sensors for some anions commonly found in the environment. The membrane sensor displayed a marked selectivity towards $\mathrm{SCN}^{-}$anions compared with other anions. The preferential response of the membrane towards $\mathrm{SCN}^{-}$anions is believed to be associated with the coordination of the $\mathrm{SCN}^{-}$anion with $\mathrm{Co}(\mathrm{II})$ metal located at the center of the CoTMeOPP ionophore. ${ }^{(12)}$

\subsubsection{Membrane composition}

The sensitivity, linear dynamic range, selectivity, and the performance of the ISEs do not depend solely on the nature of the ionophore but also depend significantly on the membrane composition. ${ }^{(24)}$ Thus, the effects of the membrane composition and the nature and amount of the lipophilic additive on the potentiometric responses of the membranes were investigated. Several membranes with different compositions were prepared and the best response was observed with the membrane composed of three layers, i.e., a first layer of Ag NPs, an inner layer of $95.14 \mathrm{wt} \%$ HEMA and $4.86 \mathrm{wt} \%$ DMPP, and an outer layer of $98.86 \mathrm{wt} \% n$-BA, 0.08 wt $\%$ HDDA, $0.34 \mathrm{wt} \%$ CoTMeOPP, $0.16 \mathrm{wt} \%$ TDDA and $0.56 \mathrm{wt} \%$ DMPP.

In Table 1, it can be seen that in the composition of the prepared self-plasticizing membrane, the ionophore acts as the active element of the membrane to detect the target analyte of $\mathrm{SCN}^{-}$(by comparing the SCN-ISE compositions with and without ionophores E1 and E3). The amount of TDDA lipophilic additive used in the membrane composition can be seen to strongly influence the selectivity and sensitivity of the self-plasticizing membrane sensor (E3 and E5). This is because the cationic additive improves the EMF response of the anion-selective electrode. ${ }^{(25)}$ For the results for E2, it can be seen that the utilization of the inner-layer self-plasticizing 
Table 1

Composition, slope, linear range, and detection limit of calibration curve for $\mathrm{SCN}^{-}$self-plasticizing Co(II)-porphyrin-based membrane GCE.

\begin{tabular}{lccccccccc}
\hline No. & $\begin{array}{c}n \text {-BA } \\
(\mu \mathrm{L})\end{array}$ & $\begin{array}{c}\text { HDDA } \\
(\mu \mathrm{L})\end{array}$ & $\begin{array}{c}\text { DMPP } \\
(\mathrm{mg})\end{array}$ & $\begin{array}{c}\text { CoTMeOPP } \\
(\mathrm{mg})\end{array}$ & $\begin{array}{c}\text { TDDA } \\
(\mathrm{mg})\end{array}$ & Working Range $(\mathrm{M})$ & $\begin{array}{c}\text { Limit of } \\
\text { detection }(\mathrm{M})\end{array}$ & $\begin{array}{c}\text { Slope } \\
(\mathrm{mV} / \mathrm{decade})\end{array}$ & $R^{2}$ \\
\hline *E0 & 950 & 1 & 1 & 0.34 & 0.8 & $1 \times 10^{-6}$ to $1 \times 10^{-1}$ & $1.02 \times 10^{-6}$ & -31.15 & 0.93 \\
E1 & 950 & 1 & 1 & 0.00 & 0.8 & $1 \times 10^{-3}$ to $1 \times 10^{-1}$ & $9.77 \times 10^{-4}$ & -22.93 & 0.97 \\
**E2 & 950 & 1 & 1 & 1.90 & 0.8 & $1 \times 10^{-7}$ to $1 \times 10^{-1}$ & $8.51 \times 10^{-8}$ & -30.83 & 0.99 \\
***E3 & 950 & 1 & 1 & 1.00 & 0.8 & $1 \times 10^{-7}$ to $1 \times 10^{-1}$ & $9.55 \times 10^{-8}$ & -60.76 & 0.99 \\
E4 & 950 & 1 & 1 & 1.90 & 0.8 & $1 \times 10^{-4}$ to $1 \times 10^{-1}$ & $9.77 \times 10^{-5}$ & -37.98 & 0.95 \\
E5 & 950 & 1 & 1 & 2.00 & 0.0 & $1 \times 10^{-4}$ to $1 \times 10^{-1}$ & $8.51 \times 10^{-5}$ & -62.69 & 0.98 \\
E6 & 950 & 1 & 1 & 2.50 & 0.8 & $1 \times 10^{-5}$ to $1 \times 10^{-1}$ & $8.32 \times 10^{-6}$ & -54.82 & 0.99 \\
E7 & 950 & 1 & 1 & 2.50 & 0.8 & $1 \times 10^{-5}$ to $1 \times 10^{-1}$ & $8.91 \times 10^{-6}$ & -65.96 & 0.97 \\
E8 & 950 & 1 & 1 & 2.50 & 0.1 & $1 \times 10^{-5}$ to $1 \times 10^{-1}$ & $8.91 \times 10^{-6}$ & -36.79 & 0.99 \\
E9 & 950 & 1 & 1 & 2.50 & 0.5 & $1 \times 10^{-4}$ to $1 \times 10^{-1}$ & $7.94 \times 10^{-5}$ & -72.87 & 0.95 \\
E10 & 950 & 1 & 1 & 2.50 & 0.7 & $1 \times 10^{-4}$ to $1 \times 10^{-1}$ & $7.08 \times 10^{-5}$ & -49.18 & 0.94 \\
E11 & 950 & 1 & 1 & 2.50 & 1.0 & $1 \times 10^{-4}$ to $1 \times 10^{-1}$ & $8.13 \times 10^{-5}$ & -50.59 & 0.95 \\
E12 & 950 & 1 & 1 & 3.00 & 0.8 & $1 \times 10^{-5}$ to $1 \times 10^{-1}$ & $9.44 \times 10^{-6}$ & -67.95 & 0.99 \\
E13 & 950 & 1 & 1 & 5.00 & 0.8 & $1 \times 10^{-5}$ to $1 \times 10^{-1}$ & $9.55 \times 10^{-6}$ & -57.48 & 0.98 \\
E14 & 950 & 1 & 1 & 7.00 & 0.8 & $1 \times 10^{-5}$ to $1 \times 10^{-1}$ & $8.32 \times 10^{-6}$ & -62.07 & 0.98 \\
\hline
\end{tabular}

*Absence of Ag NPs, **Absence of inner layer, ***Optimized composition of SCN-ISM.

conductive polymeric membranes enhanced the stability of the potential response of the sensor with significant improvement of the potential slope from -30.83 to $-60.76 \pm 0.056 \mathrm{mV} / \mathrm{decade}$ (E2 and E3). This is most probably due to the improved conductivity between the inner layer and outer layer after soaking the HEMA inner layer in KSCN solution before the deposition of the outer layer, thereby saturating the membrane with $\mathrm{SCN}^{-}$, ensuring the Nernstian slope. ${ }^{(26)}$ By comparing E0 and E3, we can see a clear improvement of the conductivity after Ag NPs are introduced to the SCN-ISE. This may be due to the enhancement in the conductivity between the target analyte and the ISE and will be explained later.

Figure 5 shows the potential slope of the optimized self-polymerized ISMs with and without the ionophore. The potential slope was significantly improved after ionophores were introduced to the SCN-ISM comprising n-BA:HDDA:DMPP:CoTMeOPP:TDDA of 98.85:0.08:0.56:0.35:0.16, $\mathrm{mol} \%$.

\subsubsection{Selectivity coefficient}

The most important characteristic of the sensors is its response towards the primary ions in the presence of other ions in the solutions, which can be expressed by the selectivity coefficient. The potentiometric selectivity coefficient $\left(K_{\mathrm{SCN}^{-}, \mathrm{M}^{-}}^{\text {pot }}\right)$ was calculated by the matched potential method (MPM) describing the selectivity toward another anion, $\mathrm{M}^{-}$, commonly found relative to $\mathrm{SCN}^{-}$in the environment. Table 2 lists the potentiometric selectivity coefficient data of the sensor for several anions relative to the $\mathrm{SCN}^{-}$anion. The results showed strong affinity of the prepared sensor to the $\mathrm{SCN}^{-}$anion compared with all other tested interfering anions since the 




Fig. 5. Potential responses of optimized SCN-ISE with and without CoTMeOPP ionophore.

Table 2

Selectivity coefficient of various anions for the proposed SCN-ISE.

\begin{tabular}{lccc}
\hline Anion & $\log K_{\mathrm{SCN}^{-}, \mathrm{M}^{-}}^{\text {pot }}$ & Anion & $\log K_{\mathrm{SCN}^{-}, \mathrm{M}^{-}}^{\text {pot }}$ \\
\hline $\mathrm{Cl}^{-}$ & -3.14 & $\mathrm{CO}_{3}{ }^{2-}$ & -2.79 \\
$\mathrm{Br}^{-}$ & -3.71 & $\mathrm{~S}_{2} \mathrm{O}_{3}{ }^{2-}$ & -2.68 \\
$\mathrm{IO}_{3}{ }^{-}$ & -4.04 & $\mathrm{SO}_{4}{ }^{2-}$ & -4.22 \\
$\mathrm{COO}^{-}$ & -4.69 & $\mathrm{~S}_{2} \mathrm{O}_{5}{ }^{2-}$ & -4.00 \\
$\mathrm{NO}_{3}{ }^{-}$ & -4.85 & $\mathrm{CrO}_{4}{ }^{2-}$ & -4.82 \\
\hline
\end{tabular}

MPM selective coefficient values were less than $-2 .{ }^{(27)}$ The selectivity pattern of the proposed $\mathrm{SCN}^{-}$-selective electrode is significantly different from the classical Hofmeister selectivity sequence and is thought to be due to the possible interaction of the anions with Co(II) metal. ${ }^{(12)}$

\subsubsection{Influence of $\mathrm{pH}$}

The effect of the $\mathrm{pH}$ of the test solution on the response of the self-plasticized membrane electrode was examined at two concentrations of $\mathrm{SCN}^{-}: 1 \times 10^{-2}$ and $1 \times 10^{-5} \mathrm{M}$. The $\mathrm{pH}$ of the test solutions was adjusted using different concentrations of hydrochloric acid $(\mathrm{HCl})$ and sodium hydroxide $(\mathrm{NaOH})$, and the results are shown in Fig. 6(a). As seen, the potentials remain constant within the $\mathrm{pH}$ range of 3-11. The significant and tremendous change in potential at $\mathrm{pH}>11$ could be related to an excessive amount of $\mathrm{OH}^{-}$anions compared with $\mathrm{SCN}^{-}$anions in the test solution, which would cause interference in the test solution as the concentration of $\mathrm{OH}^{-}$ 

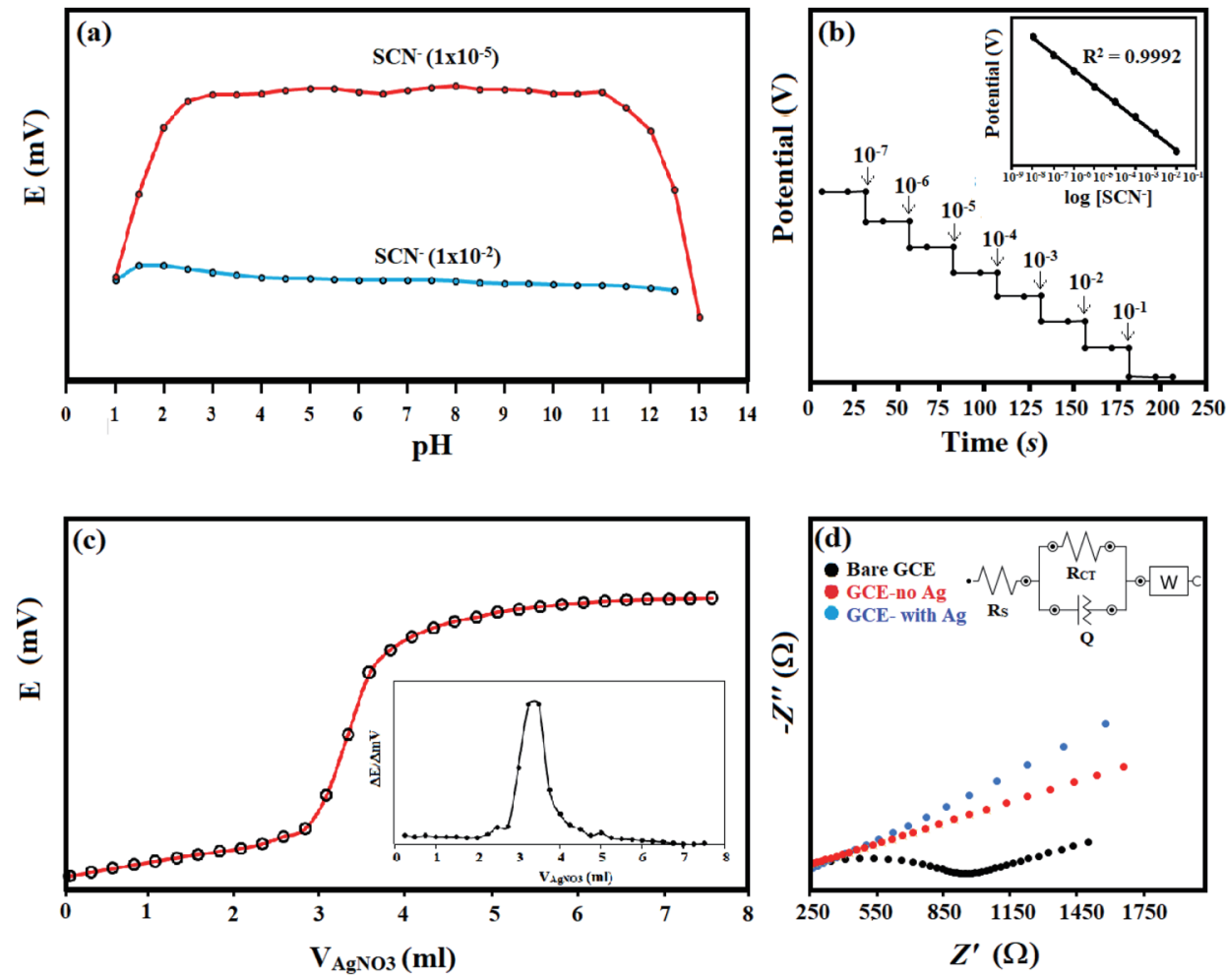

Fig. 6. (Color online) (a) Effect of $\mathrm{pH}$ on the response of the proposed SCN-ISE, (b) typical potentiometric response of optimized SCN-ISE. Inset: calibration plot. (c) Potentiometric titration curve of $25 \mathrm{ml}$ $1.52 \times 10^{-3} \mathrm{M} \mathrm{SCN}^{-}$solution with $1 \times 10^{-2} \mathrm{M} \mathrm{AgNO}_{3}$, obtained using proposed SCN-ISE. (d) Nyquist plots of bare GCE, optimized membrane without Ag, and optimized membrane with Ag layer in $0.1 \mathrm{M} \mathrm{KCl}$ solution containing $1 \mathrm{mM} \mathrm{Fe}(\mathrm{CN}) 6^{3-/ 4-}(1: 1)$.

anions is higher than that of $\mathrm{SCN}^{-}$anions. A extremely low $\mathrm{pH}$ of the test solutions $(\mathrm{pH}<3)$ may rupture the self-polymerized membranes owing to the high acidity of the test solution, thus affecting the performance of the ionophore.

\subsubsection{Repeatability and reproducibility}

The repeatability and reproducibility of the SCN-ISE were evaluated and the repeatability data shows an average slope of $-60.81 \pm 0.056 \mathrm{mV} / \mathrm{decade}$ with a relative standard deviation (RSD) of $0.092 \%$. The reproducibility data shows a fairly typical average slope of $-60.94 \pm 0.07 \mathrm{mV} /$ decade with RSD of $0.115 \%$.

\subsubsection{Lifetime}

The long-term stability of the proposed SCN-ISE was tested by performing periodic calibration with standard solutions and calculating the responses and slopes over the 
concentration ranges from $1 \times 10^{-7}$ to $1 \times 10^{-1} \mathrm{M}$. The results are presented in Table 3 . The SCN-ISE showed no significant drift when tested with $\mathrm{SCN}^{-}$standard after 14 days.

\subsubsection{Dynamic response time}

At a low concentration of $\mathrm{SCN}^{-}$solution, $\mathrm{SCN}^{-}$anions at a lower concentration are present, thus a longer time is needed for the optimized membrane to obtain a stable reading, and as the concentration of $\mathrm{SCN}^{-}$anions in the test solution increases, the time taken for the SCN-ISE to become stable decreases, as seen in Fig. 6(b). A short response time of $3 \mathrm{~s}$ was observed and recorded by changing the $\mathrm{SCN}^{-}$ion concentration in test solution from low to high.

\subsubsection{Calibration curve}

The International Union of Pure and Applied Chemistry (IUPAC) recommendation was used for the evaluation of the working concentration range and detection limit of the prepared sensor, ${ }^{(28)}$ and it was found that the linear working concentration range of the prepared SCNISE was within $1 \times 10^{-7}$ to $1 \times 10^{-1} \mathrm{M}$ with the detection limit of $9.55 \times 10^{-8} \mathrm{M}$ as shown in Fig. 5 and the Nernstian slope was $-60.76 \pm 0.056 \mathrm{mV} /$ decade.

\subsubsection{Analytical applications}

The proposed SCN-ISE was applied as an indicator electrode in the titrimetric determination of $\mathrm{SCN}^{-}$under laboratory conditions to test its applicability. The SCN-ISE was tested with a potentiometric titration of $25 \mathrm{ml}$ of $1.52 \times 10^{-3} \mathrm{M} \mathrm{SCN}^{-}$solution and $0.01 \mathrm{M} \mathrm{AgNO}_{3}$. In Fig. 6(c), it can be seen that the obtained curve is asymmetrical and the end point of the plot corresponds to the 1:1 stoichiometry of the $\mathrm{Ag}-\mathrm{SCN}$ ion complex. In the inert plot of $\Delta E / \Delta V$ vs mean volume of $\mathrm{AgNO}_{3}$, the potential demonstrated logarithmic changes with the volume of $\mathrm{AgNO}_{3}$ added and then remained almost unchanged after the end point. The obtained potential decreased with decreasing $\mathrm{SCN}^{-}$anion concentration owing to the complexation of $\mathrm{SCN}^{-}$with $\mathrm{Ag}^{+}$ions. It can be concluded that it is possible to determine the amount of $\mathrm{SCN}^{-}$anions in the solution accurately using the proposed fabricated sensor.

To evaluate the applicability of the proposed SCN-ISE in real samples, the $\mathrm{SCN}^{-}$-selective electrodes were tested with different types of sample, i.e., river water, sediment from an ex-mining lake, and saliva from both smokers and nonsmokers, as well as fish. The samples

Table 3

Linear range, detection limit, and slope of the potential response of the SCN-ISE under optimal experimental conditions.

\begin{tabular}{lccc}
\hline & Linear range $(\mathrm{M})$ & Limit of detection $(\mathrm{M})$ & Slope (mV/decade) \\
\hline Fresh & $1 \times 10^{-7}-1 \times 10^{-1}$ & $9.55 \times 10^{-8}$ & -60.76 \\
$14 \mathrm{~d}$ & $1 \times 10^{-7}-1 \times 10^{-1}$ & $6.40 \times 10^{-8}$ & -60.63 \\
$15 \mathrm{~d}$ & $1 \times 10^{-7}-1 \times 10^{-1}$ & $9.10 \times 10^{-8}$ & -59.65 \\
\hline
\end{tabular}


were pretreated to adjust the $\mathrm{pH}$ to from 4 to 10 since the proposed electrode works best at pH 3-11. The samples were analyzed in triplicate using the same electrode. The results are compared with those of IC. By analyzing the results presented in Table 4, we can see that the amount of $\mathrm{SCN}^{-}$evaluated with the proposed sensor is in good agreement with that obtained by $\mathrm{IC}$, thus reflecting the utility of the proposed sensor for detecting the $\mathrm{SCN}^{-}$anion.

Table 5 lists the linear range, detection limit, slope, response time, and interfering anions for comparative purposes, where PVC was used as the membrane in all previously reported SCN-ISE. As can be seen from the Table 5, the selectivity coefficient, linear working range, and the detection limit for the proposed electrode are superior to those of previously reported SCN-ISE with self-plasticizing membranes. This result assures that there are no leaching problems of the SCN-ISE.

Table 4

Results of applying the proposed SCN-ISE to real samples.

\begin{tabular}{lcc}
\hline Sample & IC/spiked method (M) & ISE (SCN) $(\mathrm{M})$ \\
\hline \multirow{3}{*}{ Sediments } & $8.61 \times 10^{-6}$ & $1.03 \times 10^{-5}$ \\
& $1.72 \times 10^{-5}$ & $2.07 \times 10^{-5}$ \\
& $2.58 \times 10^{-5}$ & $2.93 \times 10^{-5}$ \\
\multirow{2}{*}{ River water } & $8.61 \times 10^{-5}$ & $9.29 \times 10^{-5}$ \\
& $5.17 \times 10^{-5}$ & $6.20 \times 10^{-5}$ \\
& $3.44 \times 10^{-5}$ & $4.13 \times 10^{-5}$ \\
Fish & $6.89 \times 10^{-5}$ & $7.75 \times 10^{-5}$ \\
& $1.72 \times 10^{-4}$ & $1.82 \times 10^{-4}$ \\
& $8.61 \times 10^{-5}$ & $9.64 \times 10^{-5}$ \\
Saliva (smokers) & $1.05 \times 10^{-4}$ & $1.07 \times 10^{-4}$ \\
& $1.26 \times 10^{-4}$ & $1.45 \times 10^{-4}$ \\
& $1.02 \times 10^{-4}$ & $1.17 \times 10^{-4}$ \\
\multirow{2}{*}{ Saliva (nonsmokers) } & $3.27 \times 10^{-5}$ & $3.27 \times 10^{-5}$ \\
& $1.21 \times 10^{-6}$ & $1.72 \times 10^{-6}$ \\
& $3.44 \times 10^{-6}$ & $3.45 \times 10^{-6}$ \\
\hline
\end{tabular}

Table 5

Potentiometric parameters of the proposed $\mathrm{SCN}^{-}$-selective electrode and other $\mathrm{SCN}^{-}$-selective electrodes.

\begin{tabular}{|c|c|c|c|c|c|c|c|c|}
\hline & \multirow{2}{*}{$\begin{array}{l}\text { Proposed } \\
\text { SCN-ISE }\end{array}$} & \multicolumn{7}{|c|}{ References } \\
\hline & & (29) & (30) & (31) & (32) & (13) & (14) & (33) \\
\hline $\begin{array}{l}\text { Nernstian slope } \\
\text { (mV/decade) }\end{array}$ & -60.76 & -53 & -59.1 & -59.1 & -58.8 & -59.3 & NR & -52 \\
\hline Linear range (M) & $\begin{array}{c}1 \times 10^{-7} \text { to } \\
1 \times 10^{-1}\end{array}$ & $\begin{array}{c}1 \times 10^{-6} \text { to } \\
1 \times 10^{-1}\end{array}$ & $\begin{array}{c}1 \times 10^{-6} \text { to } \\
1 \times 10^{-1}\end{array}$ & $\begin{array}{c}1 \times 10^{-6} \text { to } \\
1 \times 10^{-1}\end{array}$ & $\begin{array}{c}1 \times 10^{-7} \text { to } \\
1 \times 10^{-2}\end{array}$ & $\begin{array}{c}1 \times 10^{-6} \text { to } \\
1 \times 10^{-1}\end{array}$ & $\begin{array}{c}1 \times 10^{-5} \text { to } \\
1 \times 10^{-1}\end{array}$ & $\begin{array}{c}1 \times 10^{-5} \text { to } \\
1 \times 10^{-1}\end{array}$ \\
\hline $\begin{array}{l}\text { Limit of } \\
\text { detection (M) }\end{array}$ & $9.55 \times 10^{-8}$ & $3.98 \times 10^{-7}$ & $5 \times 10^{-7}$ & $7 \times 10^{-7}$ & $1.25 \times 10^{-7}$ & $5 \times 10^{-7}$ & $1.9 \times 10^{-6}$ & $2.5 \times 10^{-5}$ \\
\hline Response time (s) & 3 & NR & 20 & 15 & 10 & $9-21$ & 120 & $10-15$ \\
\hline $\begin{array}{l}\text { Interfering } \\
\text { ions }\end{array}$ & - & $\mathrm{I}^{-}, \mathrm{ClO}_{4}^{-}$ & $\begin{array}{c}\text { Salicyclate, } \\
\text { I }^{-}\end{array}$ & $\mathrm{MnO}_{4}^{-}$ & $\mathrm{NO}_{3}{ }^{-}, \mathrm{I}^{-}$ & $\mathrm{I}^{-}$ & $\mathrm{Br}^{-}$ & $\mathrm{I}^{-}, \mathrm{ClO}_{4}^{-}$ \\
\hline $\mathrm{pH}$ range & $3-11$ & $1-7$ & $2-6$ & $1.5-13$ & $3-10$ & $4-10$ & NR & $2-3$ \\
\hline Lifetime (d) & 14 & NR & 30 & 60 & 90 & 60 & 7 & 120 \\
\hline
\end{tabular}

$\mathrm{NR}=$ Not reported 


\subsection{Reason for Ag NP deposition}

Ag NPs were deposited as the first layer to increase the conductivity of the $\mathrm{SCN}^{-}$-selective electrode. To study the synergistic effect between $\mathrm{Ag}$ and the self-polymerized $\mathrm{SCN}^{-}$-selective membrane, Electrochemical impedance spectroscopy (EIS) was performed to compare the internal resistance, charge transfer kinetics, and ion diffusion processes of the SCN-ISE between $100 \mathrm{kHz}$ and $1 \mathrm{mHz}$ at an $\mathrm{AC}$ potential amplitude of $5 \mathrm{mV}$. The Nyquist plots of the SCN-ISE with and without the deposition of the Ag layer in $0.1 \mathrm{M} \mathrm{KCl}$ solution containing 1 $\mathrm{mM} \mathrm{Fe}(\mathrm{CN})^{3-/ 4-}(1: 1)$ are shown in Fig. 6(d). To further analyze the behavior of the SCN-ISE, the Nyquist plots of the electrodes were fitted by a simple equivalent circuit comprising $R_{S}, R_{C T}$, $Q$, and $W$, i.e., solution resistance, charge transfer resistance, a constant phase element, and the Warburg coefficient, respectively. As can be seen, the Nyquist plots are similar to each other with a concave semicircle in the low- $Z^{\prime}(\Omega)$ region and a linear slope in the high- $Z^{\prime}(\Omega)$ region. The intersection point of the depressed concave semicircle with the real axis in the low-Z' $(\Omega)$ region represents the $R_{S}$ values. The $R_{C T}$ values were obtained from the intersection point between the semicircle diameter in the low- $Z^{\prime}(\Omega)$ region and the other semicircle diameter or linear line in the high- $Z^{\prime}(\Omega)$ region. However, a more significant decrease in $R_{C T}$ can be seen in the modified GCE $(298 \Omega, 238 \Omega)$ than in the bare GCE $(804 \Omega)$. The modified SCN-ISE with Ag NP deposition (238 $\Omega$ ) manifested the lowest $R_{C T}$ (the SCN-ISE without Ag NP deposition, $298 \Omega$; bare GCE, $804 \Omega$ ). This lowest $R_{C T}$ value is due to the high electronic conductivity attributable to the Ag NP layer deposited onto GCE. In the Nyquist plot at low frequencies, the $45^{\circ}$ slope to the real axis represents the Warburg coefficient, i.e., ion diffusion towards the electrodes. A larger Warburg coefficient manifests a longer ion diffusion path and slower ion movement. Figure 6(d) shows that the GCE modified by Ag NP deposition showed more vertical lines with higher slope, i.e., a linear plot, than the GCE without Ag NP deposition in the low- $Z$ ' $(\Omega)$ region. This indicates that the GCE modified by Ag NP deposition showed better capacity performance because of the higher electronic conductivity and faster ion diffusion of the modified GCE.

\section{Conclusions}

The results discussed in this paper indicated that a novel self-plasticized $\mathrm{SCN}^{-}$-selective membrane modified by silver NP deposition was successfully fabricated onto a GCE, and good and comparable results with that of previous SCN-ISEs in detecting $\mathrm{SCN}^{-}$anions were obtained, in which the selectivity pattern deviated from the classical Hofmeister selective pattern. Silver NPs, CoTMeOPP ionophors, HEMA, and $n$-BA self-polymerized polymers can be considered as the active ingredients in the construction of SCN-ISE self-plasticized membranes. Silver NP modification of the GCE in the fabrication of self-polymerized SCN-ISE significantly contributes enhancing the sensitivity, selectivity, and performance of the SCN-ISE. The SCN-ISE exhibits a wide working concentration range of $1 \times 10^{-1}$ to $1 \times 10^{-7} \mathrm{M}$ and a low detection limit of $9.55 \times 10^{-8} \mathrm{M}$. This sensor also shows a rapid response of $3 \mathrm{~s}$, an excellent working concentration range within $\mathrm{pHs} 3-11$, and a reasonable lifetime of 
$14 \mathrm{~d}$. The fabricated SCN-ISE was used in analytical applications for a variety of real samples of river water, ex-mine lake, human saliva, and fish. The results indicated good operating characteristics, i.e., high sensitivity, high stability, short response time, low detection limit, and wide linear working concentration range, of the SCN-ISE.

\section{Acknowledgments}

The authors gratefully acknowledge the Department of Chemistry, University of Malaya and University Malaya Centre of Ionic Liquid (UMCiL) for supporting this work. The research grant from the University Malaya Research Grant (UMRG program) (RP014A-15SUS) and Frontier Research Grant (FG030-17AFR) are also gratefully acknowledged.

\section{References}

1 E. P. Luo, Y. Q. Chai, and R. Yuan: Anal. Lett. 40 (2007) 369. https://doi.org/10.1080/00032710600964742

2 J. Zhang, C. Yang, X. Wang, and X. Yang: Anal. Bioanal. Chem. 403 (2012) 1971. https://doi.org/10.1007/ s00216-012-6002-y

3 N. Herz, S.Ferse, Y. R. Alfiansah, and A. Kunzmann: High-performance liquid chromatography to detect thiocyanate in reef fish caught with cyanide. A practical field application: Live Reef Fish (Pacific Community, 2016) pp. 8-16.

4 R. K. Bhandari, R. P. Oda, S. L. Youso, I. Petrikovics, V. S. Bebarta, G. A. Rockwood, and B. A. Logue: Anal. Bioanal. Chem. 404 (2012). https://doi.org/10.1007/s00216-012-6360-5

5 R. Sharma, M. Chhibber, and S. K. Mittal: RSC Adv. 6 (2016). https://doi.org/10.1039/C6RA03909J

6 N. R. Said, M. Rezayi, L. Narimani, N. N. Al-Mohammed, N. S. A. Manan, and Y. Alias: Electrochim. Acta 197 (2016). https://doi.org/10.1016/j.electacta.2016.02.173

7 A. R. Fakhari, M. Alaghemand, and M. Shamsipur: Anal. Lett. 34 (2001). https://doi.org/10.1081/AL100104956

8 A. R. Firooz, M.K. Amini, S. Tangestaninejad, and S. Shahrokhian: Anal. Lett. 34 (2001). https://doi. org/10.1081/AL-100103210

9 S. Sadeghi and M. Shamsipur: Anal. Lett. 33 (2000). https://doi.org/10.1080/00032710008543033

10 Z. Zhai, C. Qiu, and Y. Song: Handbook of Porphyrin Science (World Scientific, 2016) pp. 179-243. https://doi. org/10.1142/9789813149632_0003

11 K. M. Kadish and E. Van Caemelbecke: J. Solid. State. Electrochem. 7 (2003). https://doi.org/10.1007/s10008002-0306-3

12 D. Vlascici, E. Fagadar-Cosma, and O. Bizerea-Spiridon: Sensors 6 (2006). https://doi.org/10.3390/s6080892

13 A. Benvidi, M. Ghanbarzadeh, M. Dehghan, M. Mazloum-Ardakani, and R. Vafazadeh: Chin. Chem. Lett. 25 (2014). https://doi.org/10.1016/j.cclet.2014.06.020

14 F. M. Abdel-Haleem and M.S. Rizk: Mat. Sci. Eng. C 75 (2017). https://doi.org/10.1016/j.msec.2017.02.096

15 O. R. Shehab and A.M. Mansour: Biosens. Bioelectr. 57 (2014). https://doi.org/10.1016/j.bios.2014.01.051

16 W. J. Xu, Y. Q. Chai, R. Yuan, and S. L. Liu: Anal. Bioanal. Chem. 385 (2006). https://doi.org/10.1007/s00216006-0512-4

17 P. Singh and A. K. Singh: Anal. Bioanal. Chem. 400 (2011). https://doi.org/10.1007/s00216-011-4930-6

18 M. Rezayi, R. Karazhian, Y. Abdollahi, L. Narimani, S. B. T. Sany, S. Ahmadzadeh, and Y. Alias: Sci. Rep. 4 (2014). https://doi.org/10.1038/srep04664

19 L. Mendecki, X. Chen, N. Callan, D. F. Thompson, B. Schazmann, S. Granados-Focil, and A. Radu: Anal. Chem. 88 (2016). https://doi.org/10.1021/acs.analchem.5b04461

20 Y. Han, X. Wu, X. Zhang, and C. Lu: ACS Appl. Mater. Interfaces 9 (2017). https://doi.org/10.1021/ acsami.7b05204

21 M. Sookhakian, W. Basirun, M. A. M. Teridi, M. Mahmoudian, M. Azarang, E. Zalnezhad, G. Yoon, and Y. Alias: Electrochim. Acta 230 (2017). https://doi.org/10.1016/j.electacta.2017.02.022

22 R. Elder, G. J. Kennard, M. D. Payne, and E. Deutsch: Inorg. Chem. 17 (1978). https://doi.org/10.1021/ ic50183a039 
23 H. B. Silber and M. A. Murguia: Inorg. Chem. 24 (1985). https://doi.org/10.1021/ic00217a020

24 A. Abbaspour, M. Kamyabi, A. Esmaeilbeig, and R. Kia: Talanta 57 (2002). https://doi.org/10.1016/S00399140(02)00129-7

25 T. Poursaberi, M. Salavati-Niassari, S. Khodabakhsh, L. Hajiagha-Babaei, M. Shamsipur, M. Yousefi, S. Rouhani, and M. R Ganjali: Anal. Lett. 34 (2001). https://doi.org/10.1081/AL-100108409

26 Y. Qin, S. Peper, A. Radu, A. Ceresa, and E. Bakker: Anal. Chem. 75 (2003). https://doi.org/10.1021/ac0263059

27 B. Rezaei, S. Meghdadi, and S. Bagherpour: J. Hazard. Mater. 161 (2009). https://doi.org/10.1016/ j.jhazmat.2008.04.005

28 E. Lindner and Y. Umezawa: Pure Appl. Chem. 80 (2008). https://doi.org/10.1351/pac200880010085

29 D. Gao, J. Z. Li, R. Q. Yu, and G. D. Zheng: Anal. Chem. 66 (1994). https://doi.org/10.1021/ac00086a008

30 M. K. Amini, S. Shahrokhian, and S. Tangestaninejad: Anal. Lett. 32 (1999). https://doi. org $/ 10.1080 / 00032719908543002$

31 M. R. Ganjali, T. Poursaberi, F. Basiripour, M. Salavati-Niassari, M. Yousefi, and M. Shamsipur: Fresen. J. Anal. Chem. 370 (2001). https://doi.org/10.1007/s002160100915

32 S. Chandra, A. Rawat, and A. Sarkar: Anal. Lett. 41 (2008). https://doi.org/10.1080/00032710802424198

33 S. Erden, A. Demirel, S. Memon, M. Yılmaz, E. Canel, and E. Kılıç: Sens. Actuators, B 113 (2006). https://doi. org/10.1016/j.snb.2005.03.002 